\title{
Antibacterial and Anti-biofilm Efficacy of Chinese Dragon's Blood Against Staphylococcus aureus Isolated From Infected Wounds
}

\author{
Xiangkuo Zheng ${ }^{1 t}$, Lijiang Chen ${ }^{1 t}$, Weiliang Zeng ${ }^{2}$, Wenli Liao', Zhongyong Wang ${ }^{1}$, \\ Xuebin Tian'2, Renchi Fang', Yao Sun ${ }^{1}$ and Tieli Zhou't* \\ ${ }^{1}$ Department of Clinical Laboratory, The First Affiliated Hospital of Wenzhou Medical University, Wenzhou, China, ${ }^{2}$ School \\ of Laboratory Medicine and Life Sciences, Wenzhou Medical University, Wenzhou, China
}

OPEN ACCESS

Edited by:

Palanivel Velmurugan Alagappa University, India

Reviewed by: Sathiamoorthi Thangavelu, Alagappa University, India Rajakumar S.,

Bharathidasan University, India Latha Ragunathan, Aarupadai Veedu Medical College \& Hospital, India

*Correspondence:

Tieli Zhou

wyztli@163.com

${ }^{t}$ These authors have contributed equally to this work and share first

authorship

Specialty section: This article was submitted to Antimicrobials, Resistance and Chemotherapy,

a section of the journal Frontiers in Microbiology

Received: 26 February 2021 Accepted: 07 May 2021 Published: 04 June 2021

Citation:

Zheng $X$, Chen $L$, Zeng $W$, Liao W, Wang Z, Tian X, Fang R, Sun $Y$ and Zhou $T$ (2021) Antibacterial and Anti-biofilm Efficacy of Chinese

Dragon's Blood Against Staphylococcus aureus Isolated From Infected Wounds.

Front. Microbiol. 12:672943. doi: 10.3389/fmicb.2021.672943
Chinese dragon's blood (CDB), a characteristic red resin, is an important traditional Chinese medicine (TCM), and empiric therapy of infected wounds with CDB is performed in clinical settings. For the first time, we herein report the antibacterial and anti-biofilm efficacy of CDB against Staphylococcus aureus (S. aureus). Antimicrobial susceptibility testing, growth curve assay, time-kill curve assay, crystal violet biofilm assay, scanning electron microscope (SEM) analysis, cell membrane tests, and quantitative real-time polymerase chain reaction (qRT-PCR) were used for this purpose. The results suggested that the minimum inhibitory concentration (MIC) values of CDB against S. aureus ranged from 32 to $128 \mu \mathrm{g} / \mathrm{mL}$. Growth curves and time-kill curves confirmed that CDB could inhibit the growth of $S$. aureus. The biofilm formation ability and the expression levels of saeR, saeS, and hla of $S$. aureus in the presence and absence of CDB were statistically significant $(P<0.01)$. The results of SEM analysis and cell membrane tests revealed that exposure to CDB had some destructive effects on S. aureus cells. In conclusion, CDB exhibits positive antibacterial activity against S. aureus. Moreover, CDB could reduce the biofilm formation and the virulence factors of $S$. aureus by downregulating the expression levels of saeR, saeS, and hla genes. These findings indicated that CDB has immense potential to serve as a viable alternative for the treatment of infected wounds caused by S. aureus in clinical settings.

Keywords: Chinese dragon's blood, infected wounds, Staphylococcus aureus, antibacterial activity, anti-biofilm efficacy

\section{INTRODUCTION}

Worldwide, infected wounds are increasingly becoming a threat to human health (Ju et al., 2018). Acute wounds generally have a self-healing capacity and do not require significant external intervention; however, self-healing is often not possible in the case of chronic wounds. Therefore, external treatment is needed (Garcia-Villen et al., 2019). In fact, clinics face enormous challenges in managing chronic infected wounds. The normal recovery phases are altered significantly because of the presence of microbial contamination on the wound surface, leading to the possible impairment of the healing pathway and finally resulting in non-healing wounds (Garcia-Villen et al., 2019). 
Notably, Staphylococcus aureus (S. aureus) is frequently associated with infected wounds, and the pathogen is capable of biofilm formation (Salouti et al., 2016; Davis et al., 2017). Biofilms are adherent colonies of bacteria that are covered in a self-produced extracellular polysaccharide matrix (EPS) that is of host or mixed origin. Bacteria with biofilm phenotype undergo metabolic activity alterations within the protective EPS coating (Nair et al., 2016; Davis et al., 2017; Anderson et al., 2018). The biofilm enhances the ability of the organism to adapt to the environment, which in turn leads to reduced susceptibility to most antimicrobial agents. Some studies have established that alpha-toxin contributes to biofilm formation in S. aureus wound isolates (Anderson et al., 2012, 2018). Therefore, $S$. aureus biofilm formation plays a significant role in non-healing wound infections. The incorporation of antibiotics in treatment regimens has effectively eliminated multiple species of pathogens (Krychowiak et al., 2014). However, with the extensive use or even abuse of broad-spectrum antibacterial drugs, resistance to antimicrobial agents has been gradually increasing. The severe drug resistance status poses a huge challenge to anti-infective treatments in clinical settings (Song et al., 2016).

In this context, traditional Chinese medicine (TCM), including Chinese dragon's blood (CDB), has been regarded as an alternative and complementary therapeutic intervention for infected wounds (Shen et al., 2019). According to the National Drug Standard $\left[\mathrm{WS}_{3}-082(\mathrm{Z}-016)-99(\mathrm{Z})\right], \mathrm{CDB}$ is a resin with rich, deep red color, which is obtained from the fat-containing wood of the lily tree belonging to the family Liliaceae (Wang et al., 2011, 2017; Lin et al., 2020). Preclinical studies have shown that $\mathrm{CDB}$ has many phytochemicals with anti-inflammatory, antimicrobial, antifungal, and antineoplastic properties and is therefore useful in the treatment of various diseases (Pona et al., 2019). As a natural remedy for infected wounds, CDB is widely used in treating sores, diabetic foot ulcers, soft tissue injuries, etc. (Ho et al., 2016; Pona et al., 2019). Therefore, our study aimed to investigate the antibacterial and anti-biofilm efficacy of CDB against $S$. aureus isolated from infected wounds. We further attempted to provide an experimental basis for the rational use of CDB for the treatment of infected wounds in clinical settings.

\section{MATERIALS AND METHODS}

\section{Clinical Isolates and Identification}

A total of $46 \mathrm{~S}$. aureus strains were isolated from the wound specimens of the patients from the First Affiliated Hospital of Wenzhou Medical University (Zhejiang Province, China) in 2017. All strains were identified by matrix-assisted laser desorption/ionization time-of-flight mass spectrometry (MALDI-TOF MS) using the VITEK Mass Spectrometer (BioMerieux, Lyons, France). All strains were stored at $-80^{\circ} \mathrm{C}$ and incubated on blood agar plates at $37^{\circ} \mathrm{C}$ for $18-24 \mathrm{~h}$ before use.

\section{Antimicrobial Susceptibility Testing}

Antimicrobial susceptibility testing was undertaken by the agar dilution method according to the latest (Clinical and
Laboratory Standards Institute, 2020). Briefly, an overnight cultured single colony was suspended in sterile $\mathrm{NaCl}(0.85 \%)$, and the suspensions were adjusted to the turbidity equal to 0.5 McFarland standard $\left(1.5 \times 10^{8} \mathrm{CFU} / \mathrm{mL}\right)$. Then, the mixture was further diluted to $1: 10$ and evenly spotted onto the drugcontaining $\mathrm{MH}$ agar plate; the results were observed after incubation at $37^{\circ} \mathrm{C}$ for $16-18 \mathrm{~h}$. CDB (lot number: Z20O9B72911, Shanghai Yuanye Biotechnology Co., Ltd., China) was dissolved in dimethyl sulfoxide (DMSO) and tested over a range of 1$512 \mu \mathrm{g} / \mathrm{mL}$. S. aureus ATCC 29213 was employed for the quality control of the strain. The minimal inhibitory concentration (MIC) values were tested in 3 independent experiments.

\section{Bacterial Growth Curve Assay}

The bacterial growth curve was determined as previously described, with minor modifications (Zhou et al., 2018). Briefly, the 6 clinical S. aureus strains (JP-2541, JP-2718, JP-2744, JP-2850, JP-2918, and JP-3053) were isolated from patients diagnosed with different types of infected wound, as details in Supplementary Table 1. S. aureus ATCC 29213 served as the quality control strain. The 7 isolates mentioned above were cultured in fresh Luria-Bertani (LB) broth at $37^{\circ} \mathrm{C}$ with shaking at 180 revolutions per minute $(\mathrm{rpm})$ to obtain an $\mathrm{OD}_{600}$ value of 0.3 , followed by further dilution to $1: 100$ with $20 \mathrm{~mL}$ of fresh $\mathrm{LB}$ broth in the presence of $\operatorname{CDB}(16,32,64,128$, and $256 \mu \mathrm{g} / \mathrm{mL}$, respectively) as well as diluted 1:100 with fresh LB broth alone as the control group, followed subsequently by incubation at $37^{\circ} \mathrm{C}$ at $180 \mathrm{rpm}$ for overnight. The $\mathrm{OD}_{600}$ value was measured every hour for $24 \mathrm{~h}$. Therefore, a total of 24 points of data of each sample were collected. All experiments were independently repeated in triplicate.

\section{Time-Kill Curve Assay}

The time-kill curve assay for 7 S. aureus strains (JP-2541, JP2718, JP-2744, JP-2850, JP-2918, JP-3053, and ATCC 29213) was performed using a previously standardized method (Foerster et al., 2015, 2016). Briefly, overnight cultures were diluted in $20 \mathrm{~mL}$ of fresh LB broth to a final concentration of approximately $1 \times 10^{7} \mathrm{CFU} / \mathrm{mL}$, and $\mathrm{CDB}$ at $0,1 / 2 \times \mathrm{MIC}, 1 \times \mathrm{MIC}, 2 \times \mathrm{MIC}$ concentrations were added, respectively. Finally, viable colony counts were determined at $0,2,4,6,12$, and $24 \mathrm{~h}$ after incubation at $37^{\circ} \mathrm{C}$ at $180 \mathrm{rpm}$. The time-kill curves of each strain were plotted with the number of bacteria per $\mathrm{mL}(\mathrm{CFU} / \mathrm{mL})$ with the ordinates and time (h) as the abscissas.

\section{Crystal Violet Biofilm Assay}

The biofilm formation ability assay was performed according to the methods by O'Toole with some minor modifications (Niemirowicz et al., 2016). An overnight culture of each isolate was incubated at $37^{\circ} \mathrm{C} / 180 \mathrm{rpm}$ up to the logarithmic phase with an $\mathrm{OD}_{600}$ value of 0.6 , and the turbidity was adjusted to $0.5 \mathrm{McFarland}$ standard, further diluted to $1: 100$, and CDB at $0,32,64,128,256$, and $512 \mu \mathrm{g} / \mathrm{mL}$ concentrations were added, respectively. Then, $100 \mu \mathrm{L}$ of the dilution was added to the 96-well polystyrene micro-test plate (Flat bottom with lid, Sterile; Corning, United States), and 3 replicate wells were set up. Following 24 -h incubation at $37^{\circ} \mathrm{C}$ with shaking at 
$75 \mathrm{rpm}$, the upper planktonic bacteria was decanted, and biofilms attached to the well surfaces were stained with $100 \mu \mathrm{L}$ of $1 \%$ $(w / v)$ crystal violet solution (lot number: NO.20190324, Beijing Solarbio Biotechnology Co., Ltd., China) for $15 \mathrm{~min}$. The bound dye was solubilized for $30 \mathrm{~min}$ with $100 \mu \mathrm{L}$ of the eluent $(95 \%$ absolute ethanol and 5\% glacial acetic acid) and subsequently quantified by measuring the $\mathrm{OD}_{595}$ value by the Multiskan FC Microplate Reader.

\section{Scanning Electron Microscope (SEM) Analysis}

In addition to measuring the effects of CDB against $S$. aureus, SEM observation was performed, with some minor modifications (Jiamboonsri et al., 2011; Singh et al., 2017). Briefly, the prepared inoculum $(300 \mu \mathrm{L})$ of JP-2541 and S. aureus ATCC 29213 was transferred into LB broth $(2.7 \mathrm{~mL})$ in the presence and absence of $1 \times$ MIC CDB, the positively charged glass slide was placed into each well and subsequently incubated at $37^{\circ} \mathrm{C}$ overnight. The bacterial cells on the coverslip were fixed in $2.5 \%(w / v)$ of glutaraldehyde at $4^{\circ} \mathrm{C}$ for $4 \mathrm{~h}$ and rinsed with $0.1 \mathrm{M}$ of phosphate buffer ( $\mathrm{pH} 7.2)$, followed by dehydration in graded ethanol $(30,70$, and $100 \%)$ and drying at the room temperature for overnight. Finally, the dried samples were covered with gold and observed under the S-3000N scanning electron microscope (SEM) (Hitachi, Japan) at various levels of magnification.

\section{Cell Membrane Tests}

Actively growing $S$. aureus culture of each isolate was treated with serial concentrations of $\operatorname{CDB}(0,32,64,128,256$, and $512 \mu \mathrm{g} / \mathrm{mL})$ at $37^{\circ} \mathrm{C}$ for $6 \mathrm{~h}$. The alkaline phosphatase release levels of bacterial cell membrane disruption of each isolate were assessed by using a commercial kit (Solarbio, Beijing, China) (Qu et al., 2019). In an alkaline environment, AKP/ALP catalyzes the formation of phenyl disodium phosphate into free phenols. Phenols react with potassium ferricyanide and 4-aminoantipyrine to form red quinone compounds with characteristic absorbance at $510 \mathrm{~nm}$. The activity of $\mathrm{CDB}$ against $S$. aureus cell membrane was calculated by measuring the absorbance increase rate at $510 \mathrm{~nm}$.

\section{Quantitative Real-Time PCR (qRT-PCR)}

All S. aureus strains (JP-2541, JP-2718, JP-2744, JP-2850, JP2918, JP-3053, and ATCC 29213) were treated with or without $1 / 4 \times$ MIC CDB, after which the total RNA of $S$. aureus strains were extracted from the bacterial culture using the Bacterial RNA Miniprep Kit (Biomiga, Shanghai, China). The cDNA was reversed with 1000-ng RNA templates using the RevertAid First Strand cDNA Synthesis Kit (Thermo Scientific, Waltham, MA, United States). These primers are listed in Table 1 (Duan et al., 2018). The expression levels of alpha-hemolysin gene ( $h l a)$, response regulator gene $(s a e R)$, and histidine kinase gene (saeS) were analyzed by qRT-PCR. gyrb was used as an internal reference. As previously described, qRT-PCR was performed using the TB Green Premix Ex Taq II (Tli RNase H Plus) $(2 \times)$ (Takara, Japan) (Xu et al., 2020).

\section{Statistical Analysis}

The GraphPad Prism, version 8.02 (GraphPad Software, San Diego, CA, United States) was used for data analysis. The results were expressed as means $\pm \mathrm{SD}$, and comparison among the studied groups was conducted by the Student's $t$-test. Significance was considered at $P<0.05$, and all tests were two-tailed.

\section{RESULTS}

\section{Determination of MICs of CDB}

Antimicrobial susceptibility testing revealed that the MIC values of CDB against $S$. aureus ranged from 32 to $128 \mu \mathrm{g} / \mathrm{mL}$ by the agar dilution method (Table 2).

TABLE 1 | Primers used for qRT-PCR.

\begin{tabular}{ll}
\hline Primer name & Sequence $\left(\mathbf{5}^{\prime} \rightarrow \mathbf{3}^{\prime}\right)$ \\
\hline gyrb-RT-F & ACATTACAGCAGCGTATTAG \\
gyrb-RT-R & CTCATAGTGATAGGAGTCTTCT \\
hla-RT-F & TGGTAATCATCACGAACTC \\
hla-RT-R & GCAGCAGATAACTTCCTT \\
saeR-RT-F & GTCGTAACCATTAACTTCTG \\
saeR-RT-R & ATCGTGGATGATGAACAA \\
saeS-RT-F & TGTATTAAAGTGATAATATGAGTC \\
saeS-RT-R & CTTAGCCCATGATTAAAAACACC
\end{tabular}

TABLE 2 | Antibiotic susceptibility of CDB against 46 clinical S. aureus isolates and S. aureus ATCC 29213.

\begin{tabular}{lccc}
\hline Strain & CDB MIC $(\boldsymbol{\mu} \mathbf{g} / \mathbf{m L})$ & Strain & CDB MIC $(\mu \mathbf{g} / \mathbf{m L})$ \\
\hline JP-2433 & 64 & JP-2743 & 64 \\
JP-2437 & 64 & JP-2744 & 64 \\
JP-2476 & 64 & JP-2764 & 64 \\
JP-2509 & 64 & JP-2768 & 64 \\
JP-2541 & 64 & JP-2786 & 64 \\
JP-2560 & 64 & JP-2800 & 64 \\
JP-2568 & 64 & JP-2826 & 64 \\
JP-2582 & 64 & JP-2831 & 64 \\
JP-2590 & 128 & JP-2842 & 64 \\
JP-2608 & 64 & JP-2850 & 64 \\
JP-2611 & 64 & JP-2890 & 64 \\
JP-2617 & 64 & JP-2902 & 32 \\
JP-2626 & 64 & JP-2910 & 64 \\
JP-2628 & 64 & JP-2918 & 64 \\
JP-2630 & 64 & JP-2928 & 64 \\
JP-2631 & 64 & JP-2942 & 64 \\
JP-2632 & 128 & JP-2957 & 64 \\
JP-2644 & 64 & JP-2975 & 64 \\
JP-2674 & 64 & JP-3019 & 64 \\
JP-2679 & 64 & JP-3023 & 64 \\
JP-2694 & 64 & JP-3053 & 64 \\
JP-2718 & 64 & JP-3058 & 64 \\
JP-2730 & 64 & ATCC 29213 & 64 \\
JP-2738 & 64 & &
\end{tabular}




\section{Bacterial Growth Curve Assay}

By measuring the effects of $\mathrm{CDB}$ with different concentrations on the growth of $S$. aureus, it was found that CDB had no effect on the growth of $S$. aureus at the concentration $\leq 64 \mu \mathrm{g} / \mathrm{mL}$ when compared with the control group; while $\mathrm{CDB}$ with a concentration $\geq 128 \mu \mathrm{g} / \mathrm{mL}$ could effectively inhibit the growth of S. aureus (Figure 1).

\section{Time-Kill Curve Assay}

The time-kill curve assay was performed for 6 clinical S. aureus isolates (JP-2541, JP-2718, JP-2744, JP-2850, JP-2918, and JP3053) and S. aureus ATCC 29213. At $0-12 \mathrm{~h}$, our results demonstrated that all isolates kept growing in the absence of $\mathrm{CDB}$ and that all these strains remained at the initial inoculation level at the $1 / 2 \times$ MIC CDB. Interestingly, at $0-12 \mathrm{~h}$, when compared with the initial inoculum, the colony counts of the bacteria decreased by approximately 100 times at the $1 \times$ MIC CDB and were maintained at the level of the bacteria; while the growth of all strains was inhibited at $2 \times$ MIC CDB. After $12 \mathrm{~h}$, the results of this experiment revealed that $S$. aureus demonstrated a remarkable trend of increasing growth at all concentrations of $\mathrm{CDB}$ (Figure 2).

\section{Efficacy of CDB on Biofilm Formation of S. aureus}

The biofilm formation ability of $S$. aureus in the LB broth with different concentrations of CDB was compared. Our result illustrated biofilm structures of $S$. aureus in the LB broth in the presence and absence of $\mathrm{CDB}$ and noted that the differences in the biofilm formation ability between the drug-containing LB broth group and LB broth group be statistically significant $(P<0.05$; Figure 3).

\section{Scanning Electron Microscope (SEM) Analysis}

The visualization of JP-2541 and S. aureus ATCC 29213 cell morphology through SEM after treatment with CDB at $1 \times$ MIC concentration and control cells are presented in Figure 4 . The surface of $S$. aureus cells untreated with CDB (control group) formed a thick biofilm composed of aggregates and microcolonies on the coverslip at $2500 \times$ magnification, and the cell morphology was observed to be smooth with some typical characters of ball shape (Figures 4A,E). However, S. aureus cells treated with $1 \times$ MIC CDB appeared sparse and dispersed, and the number of cells was significantly reduced (Figures $4 \mathrm{~B}, \mathbf{F}$ ). Moreover, there was no change in the morphology of the control cells at $7000 \times$ magnification (Figures $\mathbf{4 C , G}$ ), whereas the CDBtreated cells showed partial destruction (Figures 4D,H).

\section{Cell Membrane Tests}

On treating these 7 isolates with different concentrations of $\mathrm{CDB}$, the differences in the release levels of alkaline phosphatase between the treated and control groups were statistically significant $(P<0.05$; Figure 5). Together, these data indicated that $\mathrm{CDB}$ could lead to increased permeability and the weakening of the cell membrane of each isolate.

\section{qRT-PCR}

The results of qRT-PCR revealed that the relative expression levels of hla and saeRS in these strains were downregulated significantly after the exposure of the $1 / 4 \times$ MIC concentration of $\mathrm{CDB}(P<0.05$; Figures $\mathbf{6 A - G})$. These results indicated that $\mathrm{CDB}$ could decrease the capacity of alpha-hemolysin production of $S$. aureus strains through the inhibition of the expression of hla, saeR, and saeS.

\section{DISCUSSION}

The skin serves as a protective physical barrier against invading microbes, including pathogens (Jun et al., 2015; Chaudhary et al., 2019). Wounds are inevitably infected by microorganisms in nature during the formation and healing processes. Infected wounds not only prolong the healing time but also threaten the lives of the patients at times (Meara et al., 2015). The pathogens responsible for the infection vary in different wound environments; nonetheless, $S$. aureus is the most common pathogen causing infected wounds (Alves et al., 2018; Rashid et al., 2019). In this scenario, it has been observed that $\mathrm{CDB}$ offers potential health benefits and could be exploited for treating infected wounds in clinical settings (Wan et al., 2019; Lin et al., 2020). Thus, we investigated the underlying antibacterial activities of $\mathrm{CDB}$ against $S$. aureus isolated from wound specimens and provided scientific evidence for antibiotic treatment of infected wounds with CDB.

To the best of our knowledge, the present study is the first to shed light on the antibacterial and anti-biofilm efficacy of CDB against $S$. aureus. Based on the results of bacterial growth curve assay and time-kill curve assay, we concluded that CDB could inhibit the growth of $S$. aureus in a concentration-dependent manner. Meanwhile, sub-inhibitory concentrations of CDB could reduce the biofilm formation ability of $S$. aureus and disrupt its cellular membrane. The above findings were further supported by SEM findings. We further uncovered that CDB could reduce the virulence factors of $S$. aureus by downregulating the expression levels of saeR, saeS, and hla and inhibiting the hemolytic activity.

$\mathrm{CDB}$ is mainly distributed in Hainan, Guangxi, and Southern Yunnan in China, and it is produced from Dracaena cochinchinensis in Indonesia, Australia, Africa, and other countries. In recent years, owing to the increasing scope of clinical application of $\mathrm{CDB}$, many scholars and clinicians have conducted in-depth research on its chemical composition and pharmacological actions. Previous studies have revealed that the main components of $\mathrm{CDB}$ are phenolics and flavonoids (Gupta and Gupta, 2011; Stefano et al., 2014; Al-Fatimi, 2018). Moreover, saponins, terpenoids, resveratrol, and other ingredients are present in it. CDB has antibacterial, anti-inflammatory, analgesic, and anti-platelet aggregation properties and is capable of promoting blood circulation and epidermal repair, besides displaying other pharmacological effects (Luo et al., 2011; Liu et al., 2013). However, little is known about the underlying antibacterial activities of $\mathrm{CBD}$ against S. aureus. Some studies have confirmed that $\mathrm{CBD}$ is rich in proanthocyanidins, phenolics, and flavonoids, which constitute 

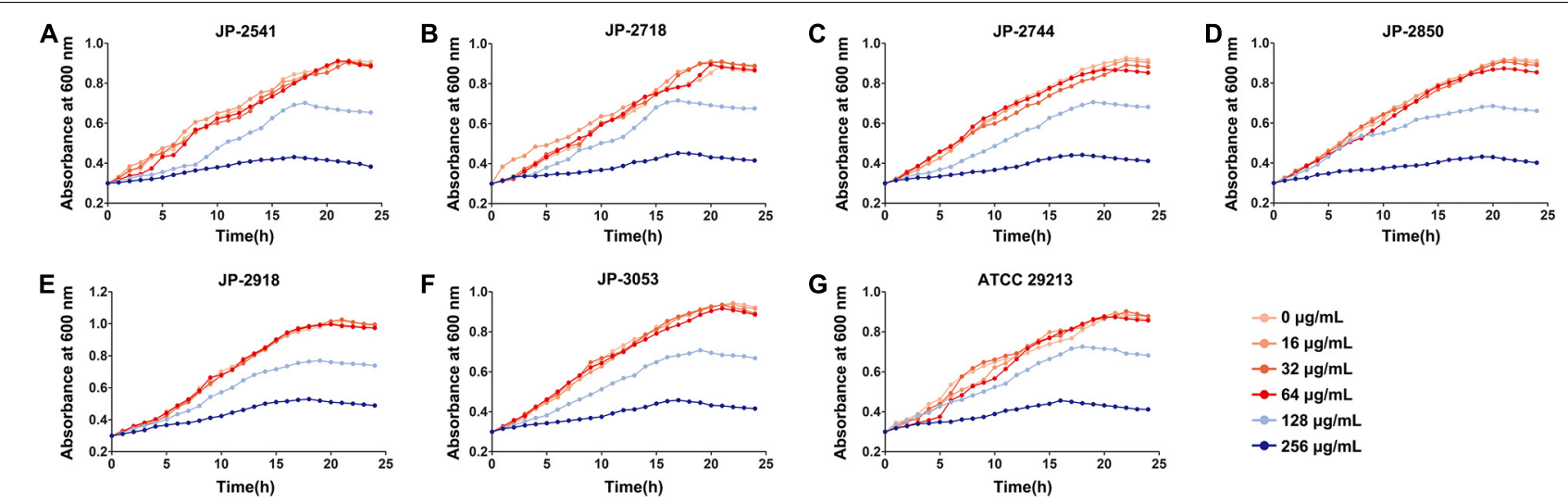

FIGURE 1 | Effects of different concentrations of CDB on the growth of Staphylococcus aureus. (A) growth curves for JP-2541; (B) growth curves for JP-2718; (C) growth curves for JP-2744; (D) growth curves for JP-2850; (E) growth curves for JP-2918; (F) growth curves for JP-3053; and (G) growth curves for S. aureus ATCC 29213.

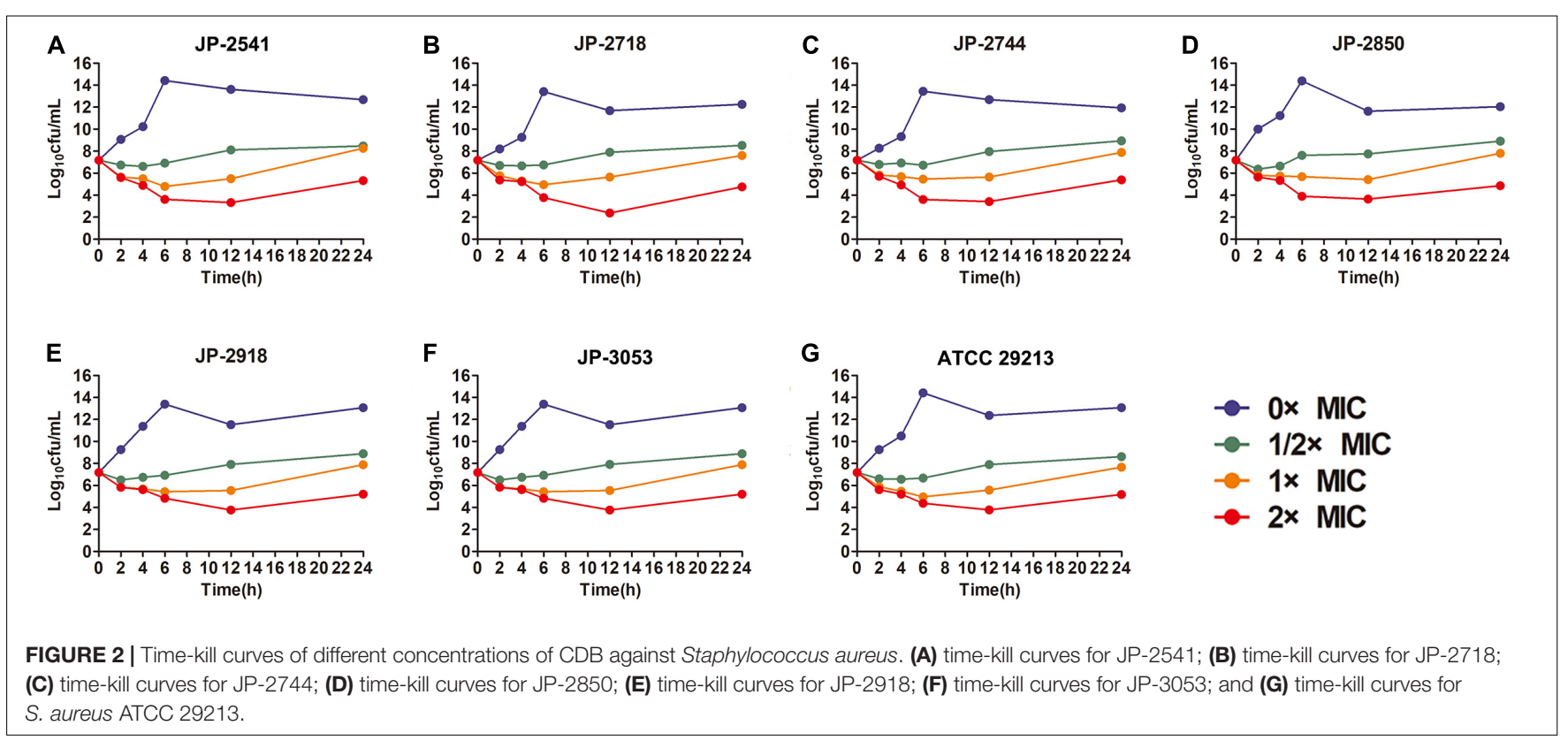

90\% of its dry weight (Jones, 2003; Al-Fatimi, 2018; Pona et al., 2019). Proanthocyanidin, as a final product of the flavonoid biosynthetic pathway, is known to effectively prevent and cure bacterial infections (Rauf et al., 2019). Also, these phenolic compounds can be easily degraded (Escobar et al., 2018). As shown in Figure 2, S. aureus exhibited remarkably increased cell growth at all concentrations of CDB after treatment for $12 \mathrm{~h}$, which is consistent with previous reports. This phenomenon reminds us that we should pay attention to such time-dependent characteristics when using $\mathrm{CDB}$ in clinical settings.

Previous studies have confirmed that in the environment of chronic infected wounds, the formation of bacterial biofilms could make the bacteria more adaptable to the external environment by increasing the adhesion to the wound surface and evading the host's immune function (Krychowiak et al., 2014). In addition, the biofilm structure could significantly enhance the pathogen's resistance to antibiotics by preventing the drugs from entering the bacterial cell, which is the main reason for persistent inflammation of the wound and difficulty in healing (van Wamel, 2017). Therefore, it is essential to find compounds that could inhibit the formation of biofilms. In our study, crystal violet biofilm assay and SEM analysis revealed that sub-inhibitory concentrations of $\mathrm{CDB}$ could effectively lower the biofilm formation ability of $S$. aureus. Biofilm formation of $S$. aureus is an important factor that determines the wound healing process and patient mortality (Bhattacharya et al., 2015; Roy et al., 2020). Besides, Tsung-Jung Ho et al. confirmed that $\mathrm{CDB}$ could stimulate angiogenesis and promote cell proliferation and migration (Ho et al., 2016). We speculated that these might be the important reasons for $\mathrm{CDB}$ promoting the wound healing process.

In addition to biofilm formation, virulence factors of S. aureus play crucial roles in wound healing either directly or indirectly. Among the various virulence determinants, alpha-hemolysin 


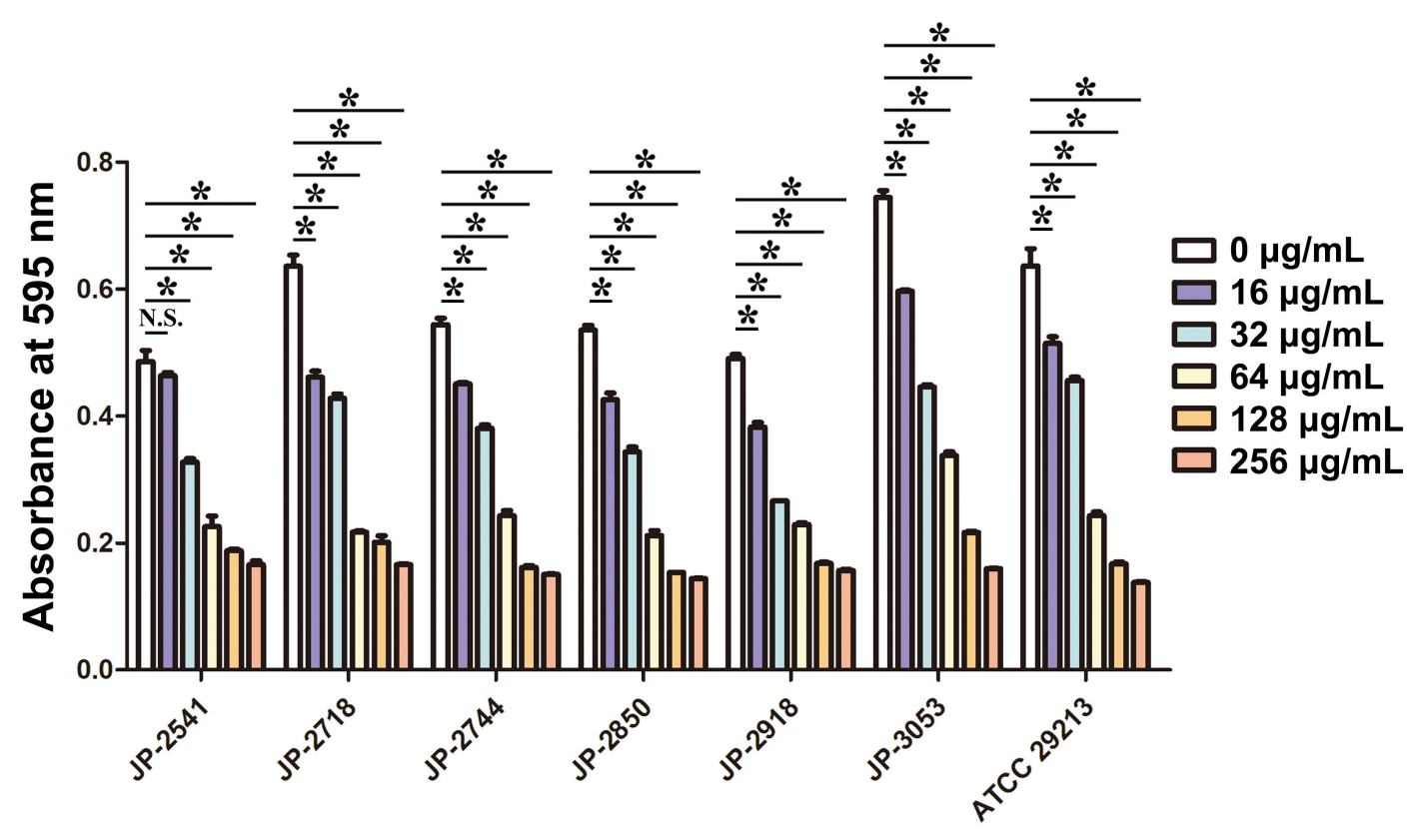

FIGURE 3 | Effects of different concentrations of CDB on the biofilm formation ability of Staphylococcus aureus. ${ }^{\star} P<0.05 ;$ N.S., $P>0.05$.

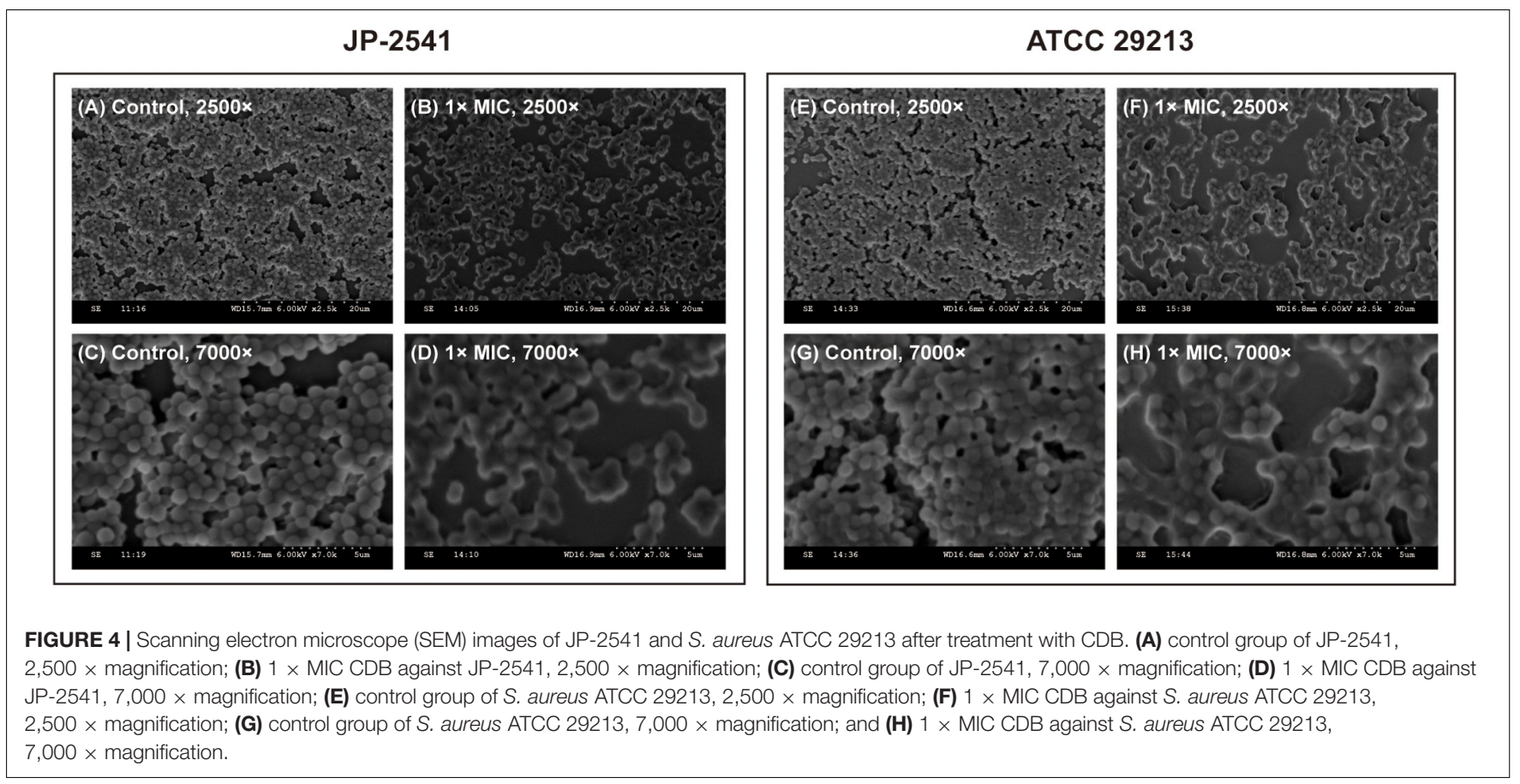

(Hla) is one of the most significant virulence factors in S. aureus wound infections, which results in attenuated production by inhibiting the expression level of the gene encoding Hla ( $h l a)$ or global regulatory genes such as saeS and saeR (Duan et al., 2018; Gudeta et al., 2019; Putra et al., 2019). The saePQRS system is a global regulator of $S$. aureus, and among them, saeS (encoding a histidine kinase) and saeR (encoding a response regulator) play regulatory roles in controlling the expression of hla (Gudeta et al., 2019; DelMain et al., 2020). As shown in qRT-PCR results, upon comparing the gene expression data of the control group with that of the group treated with $1 / 4 \times \mathrm{MIC}$ of $\mathrm{CDB}$, the transcription levels of saeR, saeS, and hla were downregulated. Our results revealed that $\mathrm{CDB}$ could decrease Hla production by $S$. aureus owing to a reduction in the expression of $s a e R$, saeS, and $h l a$, thereby potentially weakening the virulence determinants of the pathogen. 


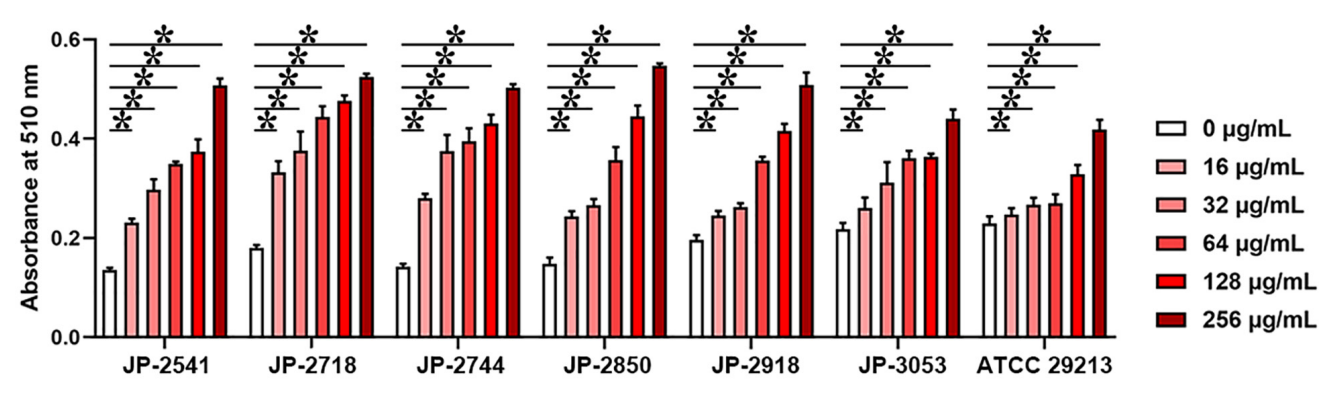

FIGURE 5 | Different concentrations of CDB used to disrupt the bacterial cell membrane integrity. ${ }^{\star} P<0.05 ;$ N.S., $P>0.05$.
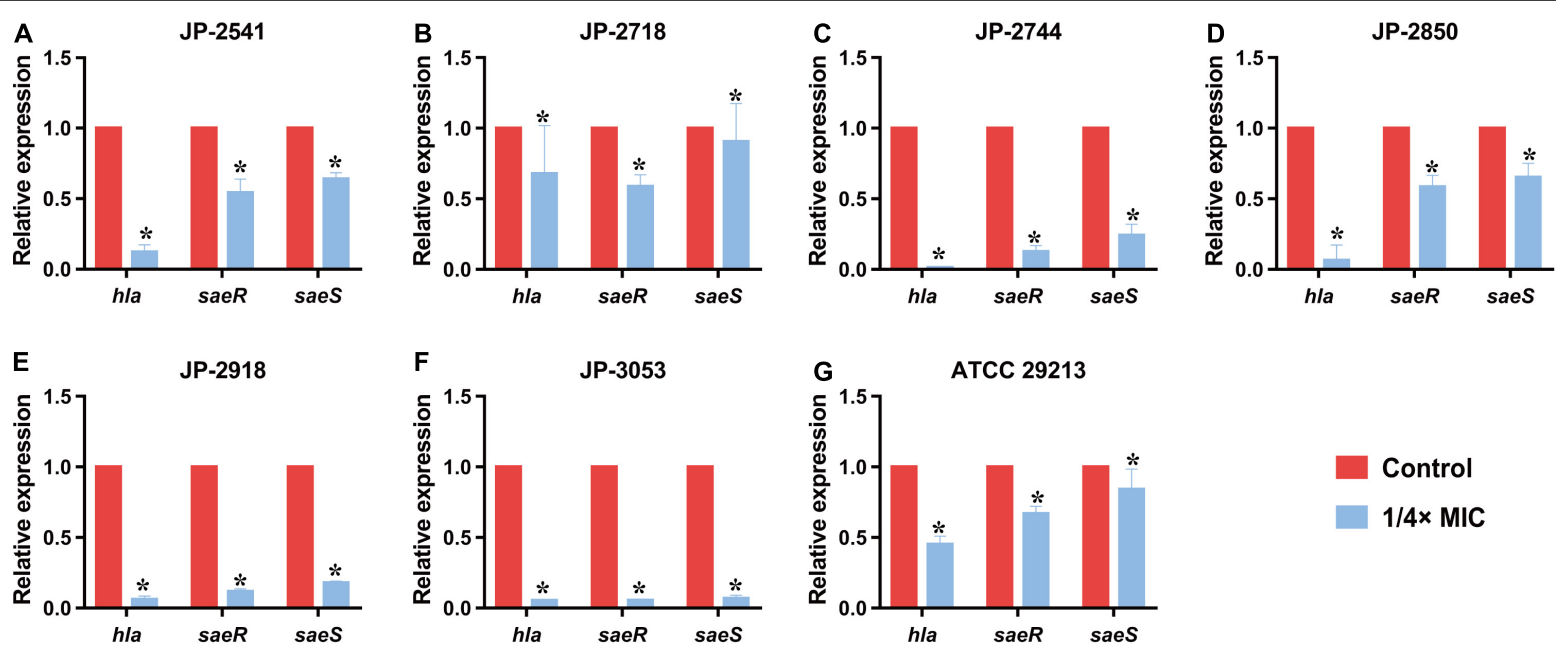

Control

$1 / 4 \times$ MIC

FIGURE 6 | Relative expression level of $h l a$, saeR, and saeS in S. aureus strains after culturing with $1 / 4 \times$ MIC concentration of CDB. ${ }^{*} P<0.05$. (A) Genes expression level in JP-2541. (B) Genes expression level in JP-2718. (C) Genes expression level in JP-2744. (D) Genes expression level in JP-2850. (E) Genes expression level in JP-2918. (F) Genes expression level in JP-3053. (G) Genes expression level in S. aureus ATCC 29213.

Therefore, we next sought to further investigate the activities of CDB against $S$. aureus in vivo by constructing the mouse model of $S$. aureus skin infected wound, and then visual observation of surface healing, bacterial counts, histology observation, and immunohistochemical analysis can be performed in the uninfected- and infected-wound groups. Most importantly, we are aware that understanding the underlying antibacterial activities of $\mathrm{CDB}$ against $S$. aureus is the crucial first step in exploring the molecular mechanisms or specific pathways of CDB to curb the growth, biofilm formation, and virulence factors of the pathogen.

\section{CONCLUSION}

In conclusion, $\mathrm{CDB}$, which is one of the most precious traditional Chinese medicine, exerts positive antibacterial efficacy on $S$. aureus, and can also reduce the biofilm formation and retard the virulence factors alpha-hemolysin of $S$. aureus by downregulating the expression levels of saeR, saeS, and hla genes. Our study provides new insights into the rational use of CDB for the treatment of infected wounds caused by $S$. aureus for the first time. These findings together indicate that CDB possesses significant potential as an alternative for the treatment of infected wounds caused by $S$. aureus in clinical settings. Furthermore, it will be worthwhile to further investigate the activities of CDB against $S$. aureus in vivo.

\section{DATA AVAILABILITY STATEMENT}

The raw data supporting the conclusions of this article will be made available by the authors, without undue reservation.

\section{ETHICS STATEMENT}

The studies involving human participants were reviewed and approved by The Ethics Committee of the First Affiliated Hospital of Wenzhou Medical University (approval number: 2021-R003). Written informed consent for participation was not required for this study in accordance with the national legislation and the institutional requirements. 


\section{AUTHOR CONTRIBUTIONS}

$\mathrm{XZ}$ and LC conducted the experiments, analyzed the data, and wrote the manuscript. WZ participated in the experiments and writing. $\mathrm{WL}, \mathrm{ZW}, \mathrm{XT}$, and $\mathrm{RF}$ analyzed the data. $\mathrm{XZ}$ and YS supervised the manuscript. TZ designed the study. All authors read and approved the final version of the manuscript for submission.

\section{FUNDING}

This study was supported by the Planned Science and Technology Project of Traditional Chinese Medicine of Zhejiang Province of China (no. 2019ZA075).

\section{REFERENCES}

Al-Fatimi, M. (2018). Ethnobotanical survey of Dracaena cinnabari and investigation of the pharmacognostical properties, antifungal and antioxidant activity of its resin. Plants (Basel) 7:91. doi: 10.3390/plants7040091

Alves, P. M., Al-Badi, E., Withycombe, C., Jones, P. M., Purdy, K. J., and Maddocks, S. E. (2018). Interaction between Staphylococcus aureus and Pseudomonas aeruginosa is beneficial for colonisation and pathogenicity in a mixed biofilm. Pathog Dis. 76:fty003. doi: 10.1093/femspd/fty003

Anderson, M. J., Lin, Y. C., Gillman, A. N., Parks, P. J., Schlievert, P. M., and Peterson, M. L. (2012). Alpha-toxin promotes Staphylococcus aureus mucosal biofilm formation. Front. Cell. Infect. Microbiol. 2:64. doi: 10.3389/fcimb.2012. 00064

Anderson, M. J., Schaaf, E., Breshears, L. M., Wallis, H. W., Johnson, J. R., Tkaczyk, C., et al. (2018). Alpha-toxin contributes to biofilm formation among Staphylococcus aureus wound isolates. Toxins (Basel) 10:157. doi: 10.3390/ toxins 10040157

Bhattacharya, M., Wozniak, D. J., Stoodley, P., and Hall-Stoodley, L. (2015). Prevention and treatment of Staphylococcus aureus biofilms. Expert Rev. Anti. Infect. Ther. 13, 1499-1516. doi: 10.1586/14787210.2015.1100533

Chaudhary, N. A., Munawar, M. D., Khan, M. T., Rehan, K., Sadiq, A., TameezUd-Din, A., et al. (2019). Epidemiology, bacteriological profile, and antibiotic sensitivity pattern of burn wounds in the burn unit of a tertiary care hospital. Cureus 11:e4794. doi: 10.7759/cureus.4794

Clinical and Laboratory Standards Institute (2020). Performance Standards for Antimicrobial Susceptibility Testing: Thirtieth Informational Supplement M100S30. Wayne, PA: CLSI.

Davis, S. C., Harding, A., Gil, J., Parajon, F., Valdes, J., Solis, M., et al. (2017). Effectiveness of a polyhexanide irrigation solution on methicillin-resistant Staphylococcus aureus biofilms in a porcine wound model. Int. Wound J. 14, 937-944. doi: 10.1111/iwj.12734

DelMain, E. A., Moormeier, D. E., Endres, J. L., Hodges, R. E., Sadykov, M. R., Horswill, A. R., et al. (2020). Stochastic expression of sae-dependent virulence genes during Staphylococcus aureus biofilm development is dependent on SaeS. mBio 11:e03081-19. doi: 10.1128/mBio.03081-19

Duan, J., Li, M., Hao, Z., Shen, X., Liu, L., Jin, Y., et al. (2018). Subinhibitory concentrations of resveratrol reduce alpha-hemolysin production in Staphylococcus aureus isolates by downregulating saeRS. Emerg. Microbes Infect. 7:136. doi: 10.1038/s41426-018-0142-x

Escobar, J. D., Prieto, C., Pardo-Figuerez, M., and Lagaron, J. M. (2018). Dragon's blood sap: storage stability and antioxidant activity. Molecules 23:2641. doi: 10.3390/molecules23102641

Foerster, S., Golparian, D., Jacobsson, S., Hathaway, L. J., Low, N., Shafer, W. M., et al. (2015). Genetic resistance determinants, In Vitro time-kill curve analysis and pharmacodynamic functions for the novel topoisomerase II Inhibitor ETX0914 (AZD0914) in Neisseria gonorrhoeae. Front. Microbiol. 6:1377. doi: $10.3389 /$ fmicb.2015.01377

\section{ACKNOWLEDGMENTS}

The authors acknowledge the financial support of the Planned Science and Technology Project of Traditional Chinese Medicine of Zhejiang Province of China (no. 2019ZA075).

\section{SUPPLEMENTARY MATERIAL}

The Supplementary Material for this article can be found online at: https://www.frontiersin.org/articles/10.3389/fmicb. 2021.672943/full\#supplementary-material

Supplementary Table 1 | Patient clinical data and characteristics of 6 clinical S. aureus isolates.

Foerster, S., Unemo, M., Hathaway, L. J., Low, N., and Althaus, C. L. (2016). Timekill curve analysis and pharmacodynamic modelling for in vitro evaluation of antimicrobials against Neisseria gonorrhoeae. BMC Microbiol. 16:216. doi: 10.1186/s12866-016-0838-9

Garcia-Villen, F., Faccendini, A., Aguzzi, C., Cerezo, P., Bonferoni, M. C., Rossi, S., et al. (2019). Montmorillonite-norfloxacin nanocomposite intended for healing of infected wounds. Int. J. Nanomedicine 14, 5051-5060. doi: 10.2147/IJN. S208713

Gudeta, D. D., Lei, M. G., and Lee, C. Y. (2019). Contribution of hla regulation by SaeR to Staphylococcus aureus USA300 pathogenesis. Infect. Immun. 87, e00231-e00319. doi: 10.1128/IAI.00231-19

Gupta, D., and Gupta, R. K. (2011). Bioprotective properties of Dragon's blood resin: in vitro evaluation of antioxidant activity and antimicrobial activity. BMC Complement Altern. Med. 11:13. doi: 10.1186/1472-6882-11-13

Ho, T. J., Jiang, S. J., Lin, G. H., Li, T. S., Yiin, L. M., Yang, J. S., et al. (2016). The In Vitro and in vivo wound healing properties of the Chinese herbal medicine "Jinchuang Ointment". Evid. Based Complement Alternat Med. 2016:1654056. doi: $10.1155 / 2016 / 1654056$

Jiamboonsri, P., Pithayanukul, P., Bavovada, R., and Chomnawang, M. T. (2011). The inhibitory potential of Thai mango seed kernel extract against methicillin-resistant Staphylococcus aureus. Molecules 16, 6255-6270. doi: 10. 3390/molecules 16086255

Jones, K. (2003). Review of sangre de drago (Croton lechleri)-a South American tree sap in the treatment of diarrhea, inflammation, insect bites, viral infections, and wounds: traditional uses to clinical research. J. Altern. Complement Med. 9, 877-896. doi: 10.1089/107555303771952235

Ju, L., McFadyen, J. D., Al-Daher, S., Alwis, I., Chen, Y., Tonnesen, L. L., et al. (2018). Compression force sensing regulates integrin alphaIIbbeta3 adhesive function on diabetic platelets. Nat. Commun. 9:1087. doi: 10.1038/s41467-01803430-6

Jun, J. I., Kim, K. H., and Lau, L. F. (2015). The matricellular protein CCN1 mediates neutrophil efferocytosis in cutaneous wound healing. Nat. Commun. 6:7386. doi: $10.1038 /$ ncomms8386

Krychowiak, M., Grinholc, M., Banasiuk, R., Krauze-Baranowska, M., Glod, D., Kawiak, A., et al. (2014). Combination of silver nanoparticles and Drosera binata extract as a possible alternative for antibiotic treatment of burn wound infections caused by resistant Staphylococcus aureus. PLoS One 9:e115727. doi: 10.1371/journal.pone.0115727

Lin, Y., Xiong, W., Xiao, S., Li, F., Lu, Z., Yan, J., et al. (2020). Pharmacoproteomics reveals the mechanism of Chinese dragon's blood in regulating the RSK/TSC2/mTOR/ribosome pathway in alleviation of DSS-induced acute ulcerative colitis. J. Ethnopharmacol. 263:113221. doi: 10.1016/j.jep.2020. 113221

Liu, H., Lin, S., Xiao, D., Zheng, X., Gu, Y., and Guo, S. (2013). Evaluation of the Wound healing potential of resina draconis (Dracaena cochinchinensis) in animal models. Evid. Based Complement Alternat. Med. 2013:709865. doi: $10.1155 / 2013 / 709865$ 
Luo, Y., Wang, H., Zhao, Y. X., Zeng, Y. B., Shen, H. Y., Dai, H. F., et al. (2011). Cytotoxic and antibacterial flavonoids from dragon's blood of Dracaena cambodiana. Planta Med. 77, 2053-2056. doi: 10.1055/s-0031-1280086

Meara, J. G., Leather, A. J., Hagander, L., Alkire, B. C., Alonso, N., Ameh, E. A., et al. (2015). Global surgery 2030: evidence and solutions for achieving health, welfare, and economic development. Surgery 158, 3-6. doi: 10.1016/j.surg.2015. 04.011

Nair, S., Desai, S., Poonacha, N., Vipra, A., and Sharma, U. (2016). Antibiofilm activity and synergistic inhibition of Staphylococcus aureus biofilms by bactericidal protein P128 in combination with antibiotics. Antimicrob. Agents Chemother. 60, 7280-7289. doi: 10.1128/AAC.0 1118-16

Niemirowicz, K., Piktel, E., Wilczewska, A. Z., Markiewicz, K. H., Durnas, B., Watek, M., et al. (2016). Core-shell magnetic nanoparticles display synergistic antibacterial effects against Pseudomonas aeruginosa and Staphylococcus aureus when combined with cathelicidin LL-37 or selected ceragenins. Int. J. Nanomed. 11, 5443-5455. doi: 10.2147/IJN.S1 13706

Pona, A., Cline, A., Kolli, S. S., Taylor, S. L., and Feldman, S. R. (2019). Review of future insights of Dragon's Blood in dermatology. Dermatol. Ther. 32:e12786. doi: $10.1111 /$ dth. 12786

Putra, I., Rabiee, B., Anwar, K. N., Gidfar, S., Shen, X., Babalooee, M., et al. (2019). Staphylococcus aureus alpha-hemolysin impairs corneal epithelial wound healing and promotes intracellular bacterial invasion. Exp. Eye Res. 181, 263-270. doi: 10.1016/j.exer.2019.02.019

Qu, S., Dai, C., Shen, Z., Tang, Q., Wang, H., Zhai, B., et al. (2019). Mechanism of synergy between tetracycline and quercetin against antibiotic resistant Escherichia coli. Front. Microbiol. 10:2536. doi: 10.3389/fmicb.2019. 02536

Rashid, A., Saqib, M., Deeba, F., and Khan, J. A. (2019). Microbial profile of burn wound infections and their antibiotic sensitivity patterns at burn unit of allied hospital Faisalabad. Pak J. Pharm Sci. 32(Suppl. 1), 247-254.

Rauf, A., Imran, M., Abu-Izneid, T., Iahtisham Ul, H., Patel, S., Pan, X., et al. (2019). Proanthocyanidins: a comprehensive review. Biomed. Pharmacother. 116:108999. doi: 10.1016/j.biopha.2019.108999

Roy, S., Santra, S., Das, A., Dixith, S., Sinha, M., Ghatak, S., et al. (2020). Staphylococcus aureus biofilm infection compromises wound healing by causing deficiencies in granulation tissue collagen. Ann. Surg. 271, 1174-1185. doi: 10.1097/SLA.0000000000003053

Salouti, M., Mirzaei, F., Shapouri, R., and Ahangari, A. (2016). Synergistic antibacterial activity of plant peptide MBP-1 and silver nanoparticles combination on healing of infected wound due to Staphylococcus aureus. Jundishapur J. Microbiol. 9:e27997. doi: 10.5812/jjm.27997

Shen, Z., Zhou, Q., Ni, Y., He, W., Shen, H., and Zhu, L. (2019). Traditional Chinese medicine for mild-to-moderate ulcerative colitis: protocol for a network meta-analysis of randomized controlled trials. Medicine (Baltimore) 98:e16881. doi: 10.1097/MD.0000000000016881

Singh, V. K., Mishra, A., and Jha, B. (2017). Anti-quorum sensing and anti-biofilm activity of delftia tsuruhatensis extract by attenuating the quorum sensingcontrolled virulence factor production in Pseudomonas aeruginosa. Front. Cell. Infect. Microbiol. 7:337. doi: 10.3389/fcimb.2017.00337

Song, Z., Sun, H., Yang, Y., Jing, H., Yang, L., Tong, Y., et al. (2016). Enhanced efficacy and anti-biofilm activity of novel nanoemulsions against skin burn wound multi-drug resistant MRSA infections. Nanomedicine 12, 1543-1555. doi: 10.1016/j.nano.2016.01.015

Stefano, V. D., Pitonzo, R., and Schillaci, D. (2014). Phytochemical and antistaphylococcal biofilm assessment of Dracaena draco L. Spp. draco resin. Pharmacogn. Mag. 10(Suppl. 2), S434-S440. doi: 10.4103/0973-1296.133300

van Wamel, W. J. B. (2017). Staphylococcus aureus infections, some second thoughts. Curr. Opin. Infect. Dis. 30, 303-308. doi: 10.1097/QCO. 0000000000000366

Wan, Y., Yu, Y., Pan, X., Mo, X., Gong, W., Liu, X., et al. (2019). Inhibition on acid-sensing ion channels and analgesic activities of flavonoids isolated from dragon's blood resin. Phytother. Res. 33, 718-727. doi: 10.1002/ptr.6262

Wang, H., Jiang, H. M., Li, F. X., Chen, H. Q., Liu, W. C., Ren, S. Z., et al. (2017). Flavonoids from artificially induced dragon's blood of Dracaena cambodiana. Fitoterapia 121, 1-5. doi: 10.1016/j.fitote.2017.06.019

Wang, X. H., Zhang, C., Yang, L. L., and Gomes-Laranjo, J. (2011). Production of dragon's blood in Dracaena cochinchinensis plants by inoculation of Fusarium proliferatum. Plant Sci. 180, 292-299. doi: 10.1016/j.plantsci.2010.09.007

Xu, W., Chen, T., Wang, H., Zeng, W., Wu, Q., Yu, K., et al. (2020). Molecular mechanisms and epidemiology of fosfomycin resistance in Staphylococcus aureus isolated from patients at a teaching hospital in China. Front. Microbiol. 11:1290. doi: 10.3389/fmicb.2020.01290

Zhou, Y., Wang, T., Guo, Y., Liu, S., Wang, J., Shen, Y., et al. (2018). In Vitro/Vivo activity of potential MCR-1 inhibitor in combination with colistin againsts $\mathrm{mcr}$ 1-Positive Klebsiella pneumonia. Front. Microbiol. 9:1615. doi: 10.3389/fmicb. 2018.01615

Conflict of Interest: The authors declare that the research was conducted in the absence of any commercial or financial relationships that could be construed as a potential conflict of interest.

Copyright (c) 2021 Zheng, Chen, Zeng, Liao, Wang, Tian, Fang, Sun and Zhou. This is an open-access article distributed under the terms of the Creative Commons Attribution License (CC BY). The use, distribution or reproduction in other forums is permitted, provided the original author(s) and the copyright owner(s) are credited and that the original publication in this journal is cited, in accordance with accepted academic practice. No use, distribution or reproduction is permitted which does not comply with these terms. 